\title{
Addition to the Article with Stepan Moskaliuk on the Inter Relationship of General Relativity and (Quantum) Geometrodynamics, via Use of Metric Uncertainty Principle
}

\section{Andrew Walcott Beckwith}

Physics Department, College of Physics, Chongqing University Huxi Campus, Chongqing, China

Email: Rwill9955b@gmail.com, abeckwith@uh.edu

How to cite this paper: Beckwith, A.W. (2016) Addition to the Article with Stepan Moskaliuk on the Inter Relationship of General Relativity and (Quantum) Geometrodynamics, via Use of Metric Uncertainty Principle. Journal of High Energy Physics, Gravitation and Cosmology, 2, 467-471.

http://dx.doi.org/10.4236/jhepgc.2016.24040

Received: July 4, 2016

Accepted: August 20, 2016

Published: August 23, 2016

Copyright (c) 2016 by author and Scientific Research Publishing Inc. This work is licensed under the Creative Commons Attribution International License (CC BY 4.0). http://creativecommons.org/licenses/by/4.0/

\begin{abstract}
We take note of the material offered in [1] as to Geometrodynamics as a way to quantify an inter relationship between a quantum style Heisenberg uncertainty principle for a metric tensor and conditions postulated as to a barotropic fluid, i.e. dust for early universe conditions. By looking at the onset of processes at/shorter than a Planck Length, in terms of initial expansion of the universe, we use inputs from the metric tensor as a starting point for the variables used in Geometrodynamics.
\end{abstract}

\section{Keywords}

General Relativity, Geometrodynamics, Metric Uncertainty Principle

\section{Introduction}

We will be using, the inputs from [1] extensively as a way to intertwine the predictions as to a HUP connected with the metric tensor of space-time and the resulting initial conditions for space-time according to Geometrodynamics. The end result will be that we are supplying initial conditions which cannot be obtained, by other means. We also will quantify via a version of dust dynamics, how this affects candidate DM and possibly DE contributions to initial cosmological conditions. To do this, we will review the concepts used in both the Heisenberg Uncertainty principle, for metric tensors, and the Geometrodynamics equations used. The conclusion of what we are talking about is use of the HUP, for metric tensors to form bounds on the Getrodynamics equations in the pre Planckian space-time era. 


\section{HUP for Metric Tensors Used}

We will be examining a Friedmann equation for the evolution of the scale factor, using explicitly two cases, one case being when the acceleration of expansion of the scale factor is kept in, another when it is out, and the intermediate cases of when the acceleration factor, and the scale factor is important but not dominant. In doing so we will be tying it in our discussion with the earlier work done on the HUP but from the context of how the acceleration term will affect the HUP, and making sense of

$$
\begin{aligned}
& \left\langle\left(\delta g_{u v}\right)^{2}\left(\hat{T}_{u v}\right)^{2}\right\rangle \geq \frac{\hbar^{2}}{V_{\text {Volume }}^{2}} \\
& \underset{u v \rightarrow t t}{\longrightarrow}\left\langle\left(\delta g_{t t}\right)^{2}\left(\hat{T}_{t t}\right)^{2}\right\rangle \geq \frac{\hbar^{2}}{V_{\text {Volume }}^{2}} \\
& \& \delta g_{r r} \sim \delta g_{\theta \theta} \sim \delta g_{\phi \phi} \sim 0^{+}
\end{aligned}
$$

Namely we will be working with

$$
\begin{aligned}
& \delta t \Delta E=\frac{\hbar}{\delta g_{t t}} \equiv \frac{\hbar}{a^{2}(t) \cdot \phi} \ll \hbar \\
& \Leftrightarrow S_{\text {initial }}\left(\text { with }\left[\delta g_{t t}\right]\right)=\left(\delta g_{t t}\right)^{-3} S_{\text {initial }}\left(\text { without }\left[\delta g_{t t}\right]\right) \gg S_{\text {initial }}\left(\text { without }\left[\delta g_{t t}\right]\right)
\end{aligned}
$$

i.e. the fluctuation $\delta g_{t t} \ll 1$ dramatically boost initial entropy. Not what it would be if $\delta g_{t t} \approx 1$. The next question to ask would be how could one actually have

$$
\delta g_{t t} \sim a^{2}(t) \cdot \phi \underset{\phi \sim \text { Very Large }}{\longrightarrow} 1 .
$$

In short, we would require an enormous "inflaton" style $\phi$ valued scalar function, and $a^{2}(t) \sim 10^{-110}$ How could $\phi$ be initially quite large? Within Planck time the following for mass holds, as a lower bound

$$
m_{\text {graviton }} \geq \frac{2 \hbar^{2}}{\left(\delta g_{t t}\right)^{2} l_{P}^{2}} \cdot \frac{(E-V)}{\Delta T_{t t}^{2}} .
$$

Here, we are using the following approximation as to Kinetic energy in the beginning of the expansion of the universe.

$$
\text { K.E. } \sim(E-V) \sim \dot{\phi}^{2} \propto a^{-6}
$$

Then, up to first order, we could approximate, with H.O.T. being higher order terms

$$
\dot{\phi} \sim a^{-3} \Leftrightarrow \phi \approx t \cdot a^{-3}+\text { H.O.T } .
$$

This Equation (6) will be considerably refined in the subsequent document.

\section{The Role of Geometrodynamics. And How Equation (2) May Be Applied}

From Equation (48) of [1] we have 


$$
\begin{aligned}
& \left\langle u_{k}\left|H_{\phi}\right| u_{k^{\prime}}\right\rangle=M_{k}(a) \cdot \delta_{k, k^{\prime}} \\
& \underset{V(\phi)=\lambda_{\alpha} \phi^{\alpha} \& k=k^{\prime}}{\longrightarrow} \varepsilon_{k} \cdot\left(\frac{\lambda_{\alpha}}{2}\right)^{\left(\frac{2}{2+\alpha}\right)} a^{\left(\frac{3 \cdot(2-\alpha)}{2+\alpha}\right)} \\
& \underset{\alpha=2}{\longrightarrow}\left\langle u_{k}\left|H_{\phi}\right| u_{k^{\prime}}\right\rangle=M_{k}(a) \cdot \delta_{k, k^{\prime}} \\
& \underset{V(\phi)=\lambda_{\alpha} \phi^{\alpha} \& k=k^{\prime}}{\longrightarrow} \varepsilon_{k} \cdot\left(\frac{\lambda_{\alpha}}{2}\right)^{\left(\frac{2}{2+\alpha}\right)} a^{\left(\frac{3 \cdot(2-\alpha)}{2+\alpha}\right)} \\
& \underset{\alpha=2}{\longrightarrow} \sqrt{2 \lambda_{2}} \cdot(k+1 / 2)=M_{2}(a)
\end{aligned}
$$

Here, we can also, from [1] assign a density functional and then a change of energy as given by $\Delta E=2 \cdot 10^{-\gamma} \cdot l_{P}^{3} M_{2}(a) / a^{3}$. So then, that one will have

$$
\begin{aligned}
& \rho_{m}=2 M_{2}(a) / a^{3}=\sqrt{2^{3} \cdot \lambda_{2}} \cdot a^{-3} \cdot(k+1 / 2) \\
& \Delta E=2 \cdot 10^{-\gamma} \cdot l_{P}^{3} M_{2}(a) / a^{3}=10^{-\gamma} \cdot l_{P}^{3} \cdot \sqrt{2^{3} \cdot \lambda_{2}} \cdot a^{-3} \cdot(k+1 / 2)
\end{aligned}
$$

This change of energy will then be put into Equation (2) with the result that.

Here the subscript k, as in Equation (8) is by [1] a "particle count" and we will refer to this heavily in the rest of this document. If we have Equation (8) we will, if we have an emergent field reference using a change in energy, in the Pre Planckian domain as

$$
\delta g_{t t} \approx \frac{\hbar}{\delta t \Delta E}=(\delta t)^{-1} \cdot \frac{\hbar}{10^{-\gamma} \cdot l_{P}^{3} \cdot \sqrt{2^{3} \cdot \lambda_{2}} \cdot a^{-3} \cdot(k+1 / 2)} .
$$

Or, if the inequality is strictly adhered to

$$
\delta g_{t t} \geq \frac{\hbar}{\delta t \Delta E}=(\delta t)^{-1} \cdot \frac{\hbar}{10^{-\gamma} \cdot l_{P}^{3} \cdot \sqrt{2^{3} \cdot \lambda_{2}} \cdot a^{-3} \cdot(k+1 / 2)} .
$$

The smallness of the initial scale factor would be of the order of $a^{-3} \sim 10^{165}$, and we have that $k \sim 10^{20}$, initially, and that $l_{P}^{3} \sim 10^{-105}$, and we pick $\hbar \doteq 1$ dimensionally, so then if $\delta t \sim 10^{-44}$, we have if we use Equation (9) as an estimator, that the following has to be done to insure in pre Planckian space time, for the following to hold:

$$
\begin{aligned}
& \lambda_{2} \leq 10^{-74+2 \gamma} \Leftrightarrow \delta g_{t t} \leq 1 \Leftrightarrow \delta t \Delta E \geq 1 \\
& \& \lambda_{2}>10^{-74+2 \gamma} \Leftrightarrow \delta g_{t t}>1 \Leftrightarrow \delta t \Delta E<1
\end{aligned}
$$

i.e. the violation of an uncertainty principle for commences for any situation which implies restraints on $\lambda_{2} \leq 10^{-74+2 \gamma} \Leftrightarrow \delta g_{t t} \leq 1 \Leftrightarrow \delta t \Delta E \geq 1$ when

$$
\lambda_{2}>10^{-74+2 \gamma} \Leftrightarrow \delta g_{t t}>1 \Leftrightarrow \delta t \Delta E<1 .
$$

For the problem represented by Equation (10a) to hold it would mean that the following Pre-Planckian Potential energy would be then small when the following Potential energy as given in Equation (11) is much smaller than the Kinetic energy given in Equation (2)

$$
V(\phi)=\lambda_{\alpha} \phi^{\alpha}=\lambda_{2} \phi^{2} .
$$

From inspection, for Equation (11) to hold, for our physical system we would want Equation (10) to hold which would mean an extremely small Potential energy, as op- 
posed to the large value of the Kinetic energy given in Equation (4). Hence the role of Geometrodynamics given in Equation (7) and Equation (8), will in the case of a quartic potential imply that Equation (11) as Potential energy is much smaller than the kinetic energy as represented for Pre Planckian space-time physics.

\section{Discussion and Conclusion}

What we are doing is confirming the material given in [1] as well as giving an explanation for [2].

The potential used, the quartic, is the simplest version of the potential systems in [1] and the cases of non quartic potential should be examined fully, as part of a comprehensive study. This will be part of the research project which the authors will initiate in future publications. We should keep this discussion and the discussion of scalar fields separate from the ideas given in inflation, namely of the fluctuations not necessarily having an upper bound of

$$
\tilde{\tilde{\phi}}>\sqrt{\frac{60}{2 \cdot \pi}} M_{P} \approx 3.1 M_{P} \equiv 3.1
$$

Since our modeling is not predicated upon the inflationary model of cosmology but which is addressing the issue brought up in [2], which is the contribution of Pre Planckian space time to cosmological evolution we wish to adhere to non inflationary treatments as to Equation (11) and Equation (12) but will adhere to the questions poised at the beginning of this document. Furthermore we will adhere to, in future documents in delineating a departure from the standard treatment of the evolution of the scalar field, as given in conventional inflation cosmology as the following

$$
\frac{\mathrm{d} \phi}{\mathrm{d} t}=-\frac{V^{\prime}(\phi)}{3 H(\phi)}+\frac{H^{3 / 2}(\phi)}{2 \pi} \cdot \xi(t)
$$

This has a quasi "quantum mechanical" effective white noise introduced term $\xi(t)$, and is similar to $\xi(t)$ in a first order differential equation being a "driving" term to a quasi chaotic oscillatory behavior to the scalar field. We argue that this Equation (13) in [3] is wrong, albeit well motivated by conventional inflationary cosmology and part of our future discussion will be in, for the Pre Planckian regime of space time as partly brought up in [4] discussing what we are putting in instead as a replacement. This Equation (13) contravenes our description of Kinetic energy as the dominant term in Pre Planckian space-time physics which deserves future developments for establishing experimental measurements.

\section{Acknowledgements}

This work is supported in part by National Nature Science Foundation of China grant No. 11375279.

\section{References}

[1] Text of Original Article by A. Beckwith, and Stepan Moskaliuk. 
[2] Beckwith, A. (2016) Gedanken Experiment for Fluctuation of Mass of a Graviton, Based on the Trace of GR Stress Energy Tensor-Pre Planckian Conditions That Lead to Gaining of Graviton Mass, and Planckian Conditions That Lead to Graviton Mass Shrinking to $10^{-62}$ Grams. Journal of High Energy Physics, Gravitation and Cosmology, 2, 19-24. http://dx.doi.org/10.4236/jhepgc.2016.21002

[3] Meszhlumian, A. (1992) Towards the Theory of Stationary Universe. In: Akerlof, C. and Srednicki, M., Eds., Texas/PASCOS 92: Relativivistic Astrophysics and Particle Cosmology, Annals of the New York Academy of Sciences, Vol. 688, 464-471.

[4] Beckwith, A. (2016) Gedanken Experiment Examining How Kinetic Energy Would Dominate Potential Energy, in Pre-Planckian Space-Time Physics, and Allow Us to Avoid the BICEP 2 Mistake. Journal of High Energy Physics, Gravitation and Cosmology, 2, 75-82. http://dx.doi.org/10.4236/jhepgc.2016.21008

\section{Submit or recommend next manuscript to SCIRP and we will provide best service} for you:

Accepting pre-submission inquiries through Email, Facebook, LinkedIn, Twitter, etc. A wide selection of journals (inclusive of 9 subjects, more than 200 journals)

Providing 24-hour high-quality service

User-friendly online submission system

Fair and swift peer-review system

Efficient typesetting and proofreading procedure

Display of the result of downloads and visits, as well as the number of cited articles Maximum dissemination of your research work

Submit your manuscript at: http://papersubmission.scirp.org/ 